\title{
Energy Dependence of Delayed-Neutron Data
}

\author{
Daniela FOLIGNO ${ }^{1, *}$ and Pierre LECONTE ${ }^{1, * *}$ \\ ${ }^{1}$ CEA, DEN, DER/SPRC/LEPh Cadarache, F-13108 Saint Paul-Lez-Durance, France
}

\begin{abstract}
Delayed neutron data is essential in inherent reactor safety and in reactor control. The different sources and results of delayed neutron data in international databases will be discussed. In addition to direct measurement, these data can be obtained by calculations based on fission yields (FY) and radioactive decay data (RDD). Unfortunately, FY are only given at three energies in the European library JEFF. This works proposes to use GEF-FY energy dependence to extrapolate JEFF-FY at other energies. The preliminary results highlight that GEF energy dependence is not always suitable to reproduce experimental values and that more measurements are needed at high energies.
\end{abstract}

\section{Introduction}

A DN (Delayed Neutron) is a neutron which is not created by the fission event itself but appears during the de-excitation of fission products. For example, ${ }^{137} \mathrm{I}$ is an unstable neutronrich FP (Fission Product) ${ }^{1}$ and it undergoes $\beta^{-}$-decay, leading to ${ }^{137} \mathrm{Xe}$. Depending on the excitation energy of the Xenon, and on the separation energy of the last neutron, the Xenon might emit a neutron or a gamma ray, both of which will be called delayed because they appear some time after the fission event. In nuclear physics Iodine is considered the DN precursor. In reactor applications it is extremely important to have a good estimate of the reactivity, because it tells how far the system is from criticality. The Nordheim equation (Eq. 1) allows to compute the reactivity $(\rho)$ from a measurable quantity, the reactor period $(T)[1$, and references therein].

$$
\begin{gathered}
\rho=\frac{\Lambda}{T}+\beta_{e f f} \sum_{i=1}^{G} \frac{a_{i}}{1+\lambda_{i} T} \approx \beta_{e f f} \frac{\overline{T_{1 / 2}}}{T} \\
\beta_{e f f}=\frac{\sum_{k} \sum_{i=1}^{G} \int_{0}^{\infty} a_{k, i} v_{d, k}(E) \Sigma_{f, k}(E) \Phi(E) \mathrm{d} E \int_{0}^{\infty} \chi_{d, k, i}\left(E^{\prime}\right) \Phi^{+}\left(E^{\prime}\right) \mathrm{d} E^{\prime}}{\sum_{k} \int_{0}^{\infty} a_{k, i} v_{t, k}(E) \Sigma_{f, k}(E) \Phi(E) \mathrm{d} E \int_{0}^{\infty} \chi_{t, k}\left(E^{\prime}\right) \Phi^{+}\left(E^{\prime}\right) \mathrm{d} E^{\prime}} \\
\overline{T_{1 / 2}}=\sum_{i=1}^{G} \frac{a_{i}}{\lambda_{i}}
\end{gathered}
$$

\footnotetext{
*e-mail: daniela.foligno@cea.fr

**e-mail: pierre.leconte@cea.fr

${ }^{1}$ It is considered here that the prompt neutron emission had already taken place
} 
In Eq. $1 \Lambda$ is the prompt neutron generation time, $\beta_{\text {eff }}$ the effective delayed neutron fraction, $a_{i}$ and $\lambda_{i}$ the kinetic parameters in the group approximation and $\overline{T_{1 / 2}}$ the mean precursors' half-life. All except $\Lambda$ are delayed neutrons parameters. $\beta_{\text {eff }}$ represents the fractions of neutrons that caused fission and that were born delayed. In Eq. 2 [6], $\Phi$ and $\Phi^{+}$ are the direct and the adjoint neutron flux, $\Sigma_{f, k}(E)$ the macroscopic fission cross section of the fissioning system $k, \chi_{t, k}$ the prompt neutron spectrum of $k, \chi_{d, k, i}$ the DN spectrum of $k$ per DN group $i, v_{t, k}$ and $v_{d, k}$ the prompt and delayed neutron yields, respectively. The $\overline{T_{1 / 2}}$ represents the mean time after which half of the DN have been emitted and can be computed if the kinetic parameters are known. Equations 2 and 3 show how to compute $\beta_{\text {eff }}$ and $\overline{T_{1 / 2}}$ and are derived from [2]. It is evident the need of mastering the energy dependence of those parameters, especially in fast reactors where the incident neutron energy ranges from some $\mathrm{keV}$ to some $\mathrm{MeV}$.

In section 2 the state of the art on delayed neutron data is recalled, together with the history of the libraries updates. Section 3 the methods used to compute the different quantities of interest are introduced. Furthermore, the technique employed to apply the GEF energy dependence to JEFF-3.1.1 FY is illustrated. The results are discussed in section 4 and the conclusions and perspectives in section 5 .

\section{State of the art}

The average delayed neutron yield as a function of the incident neutron energy can be found in the evaluated libraries $(\mathrm{MF}=1, \mathrm{MT}=455)$.

The origin of the recommended data is not always well defined. According to the references reported in the header of the file, the yields contained in the version 2.2 of the European Library JEFF, come from a semi-empirical calculation performed by Eric Fort using Lendel's model [3]. In the following version, JEFF-3.0, the yields have the reference England89 [4], the same of the American Library ENDF/B-VI. Overlapping the two sets of data confirmed the coherence of the references. From JEFF-3.1 to JEFF-3.1.2, the reference stayed unchanged, even though a comparison of the curves (see Fig. 1a) shows a different energy dependence. The last two versions, JEFF-3.2 and JEFF-3.3 recursively refer to the previous versions, thus not revealing the origin of the yields reported from JEFF-3.1 on. Following researches led to the conclusion that the mysterious set of data comes from TENDL2009 and that the reference in the header of the file is wrong and misleading.

As far as the American library is concerned, the last version (ENDF/B-VIII.0) takes the $v_{d}$ from the previous ENDF/B-VII.1, which in turn took it from ENDF/B-VI, "except between 1.0-5 eV and $50 \mathrm{keV}$ where they were modified to agree with JENDL-3.3 values, following a suggestion from C. Lubitz [Lu06]" [5, ENDF/B-VII.1 library, MT=451]. Finally, ENDF/B-VI seems to have taken the yields from the already mentioned England89 [4].

Another way to obtain the average delayed neutron yield as a function of the incident neutron energy is to take the fission yields at different energies and to use them, together with a radioactive decay data set, in a summation calculation performed by the author (see the method in Section 3). Figure 1 shows the $v_{d}$ as given by JEFF-3.3 (1a) and ENDF/B-VII.1 (1b), but also what it is obtained when using JEFF-3.1.1 FY and JEFF-3.1.1 or ENDF/BVIII.0 RDD (1c). Since the FY are only given at three energies (thermal, fast and high), only three points can be drawn, and nothing can be inferred on the energy dependence. 


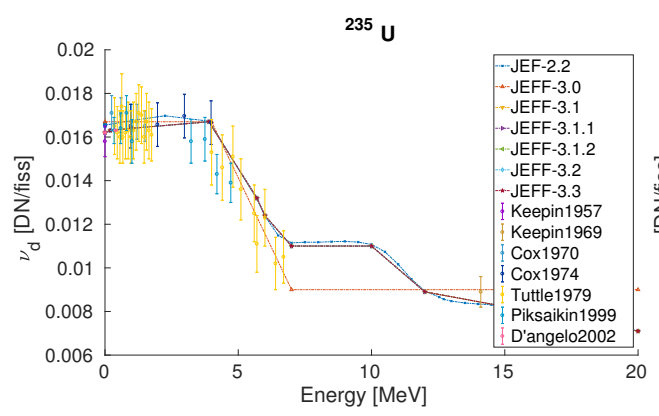

(a) JEFF library

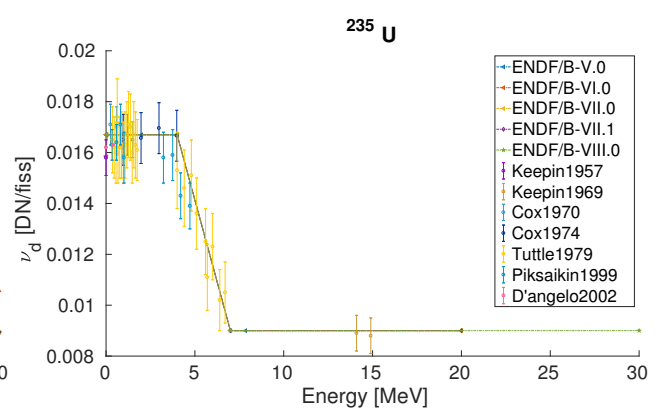

(b) ENDF/B library

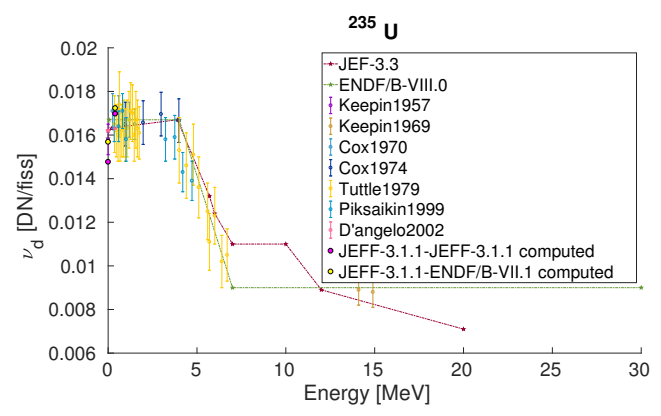

(c) Summation calculation

Figure 1: Library comparison of the average delayed neutron yield as a function of energy for ${ }^{235} \mathrm{U}$. Fig. 1a contains all the versions of JEFF library from the 2.2 to the 3.3. Fig. $1 \mathrm{~b}$ contains all the versions of JEFF library from the V.0 to the VIII.0. Fig. 1c contains JEFF3.1.1, ENDF/B-VII.1 and the points obtained with a summation calculation. All figures show experimental data for comparison.

\section{Method}

One way of estimating integral delayed-neutron quantities is to perform a summation calculation, in which the microscopic contribution of each delayed-neutron precursor is taken into account and summed up. Equation 4 shows that the contribution of a precursor to the average DN yield is the product of its cumulative fission yield $C Y$, its DN emission probability $P_{n}$ and the number of neutrons emitted per decay $x$.

$$
v_{d}=\sum_{j}^{N} C Y_{j} P_{n, j} x_{j}
$$

In the same fashion, Eq. 5 is used to compute the DN activity. In this case, instead of cumulative fission yields one has to take into account the concentration of each precursor at each time $t$. The system of Bateman differential equations has to be solved in a consistent way, in order to take into account both the precursors created by direct fission and the ones created by the decay of other fission products. 


$$
\frac{n_{d}(t)}{n_{d}\left(t_{\infty}\right)}-\sum_{i=1}^{G}\left(1-\sum_{\substack{j=1 \\ j \neq i}}^{G} a_{j}\right)\left(1-e^{-\lambda_{i} t_{i r r}}\right) e^{-\lambda_{i}\left(t-t_{i r r}\right)}=0
$$

Equation 5 shows one way to compute the kinetic parameters from the delayed neutron activity curve. The method consists at fitting the difference of two models. The first term is the normalized emission rate computed by summation calculation while the second term is the sum of exponentials we want to fit the curve with. $G$ is the number of DN groups and $a_{i}$ and $\lambda_{i}$ are the abundance and decay constant of the DN group $i$. Note that the fact of computing $a_{i}$ as $1-\sum_{\substack{j=1 \\ j \neq i}}^{G} a_{j}$ is done to normalize to 1 the sum of the abundances without introducing any normalization factor, which would have had the effect of increasing both the uncertainty and the correlations among abundances. With the DN activity (measured or calculated), the kinetic parameters and the mean precursors' half-life are easy to estimate (see Eq. 5 and Eq. 3).

GEF [7] is a Monte Carlo code able to provide FY at almost any neutron energy. When used in a calculation, the resulting $v_{d}$ are pretty far from the recommended values. This work aims at testing the energy dependence of GEF fission yields, which will be used to normalize the data of the JEFF library. First of all, one has to choose a reference energy $\left(E_{r e f}\right)$ per fissioning system, at which JEFF fission yields will be taken $\left(F Y_{J E F F}\left(E_{r e f}\right)\right)$. In this work the reference energy is $0.025 \mathrm{meV}$ for ${ }^{235} \mathrm{U}$ and ${ }^{239} \mathrm{Pu}$ and $400 \mathrm{keV}$ for ${ }^{238} \mathrm{U}$. For each point in energy, the ratio of GEF fission yields at $E$ and at $E_{r e f}$ is computed. The last step consists at applying the GEF energy dependence - the ratio $\frac{F Y_{G E F}(E)}{F Y_{G E F}\left(E_{r e f}\right)}$ - to the reference $F Y_{J E F F}\left(E_{r e f}\right)$ (see Eq. 6).

$$
F Y_{J E F F}(E)=\frac{F Y_{G E F}(E)}{F Y_{G E F}\left(E_{r e f}\right)} F Y_{J E F F}\left(E_{r e f}\right)
$$

The new sets of JEFF fission yields at different energies are then coupled with ENDF/BVIII.0 radioactive decay data to perform a summation calculaton through which the quantities of interest can be estimated (Eq. 4 and 5).

\section{Results}

\subsection{Average delayed-neutron yield}

The results of the calculation and the comparison with experimental data are shown in Fig. 2, where $2 \mathrm{a}, 2 \mathrm{~b}$ and $2 \mathrm{c}$, refer to ${ }^{235} \mathrm{U},{ }^{238} \mathrm{U}$ and ${ }^{239} \mathrm{Pu}$, respectively. The RDD is taken from ENDF/B-VIII.0. In the three plots the blue line corresponds to the summation calculation performed by using GEF-6.1 FY, while the orange one corresponds to JEFF-3.1.1 FY, corrected with GEF-6.1 energy dependence.

As far as the ${ }^{238} \mathrm{U}$ is concerned, the shape of the orange curve, with a well-defined stepwise behavior, is promising. Furthermore the agreement with the experimental data, especially below $10 \mathrm{MeV}$, is reassuring. Applying GEF energy dependence to JEFF fission yields for ${ }^{235} \mathrm{U}$ and ${ }^{239} \mathrm{Pu}$, an unexpected bump appears around 16-17 MeV. Moreover, the expected flat behavior below $4 \mathrm{MeV}$ as well as the experimental slope for ${ }^{235} \mathrm{U}$ are not reproduced by the calculation. Unfortunately, in the intermediate range (7 to $13 \mathrm{MeV}$ ) a lack of measurements prevents the author draw any conclusion. 


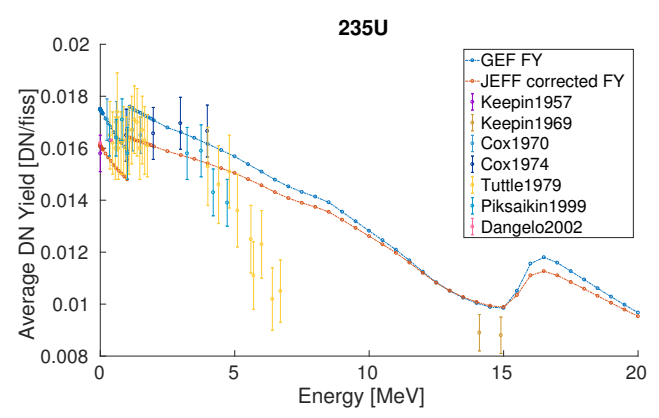

(a) $235 \mathrm{U}$

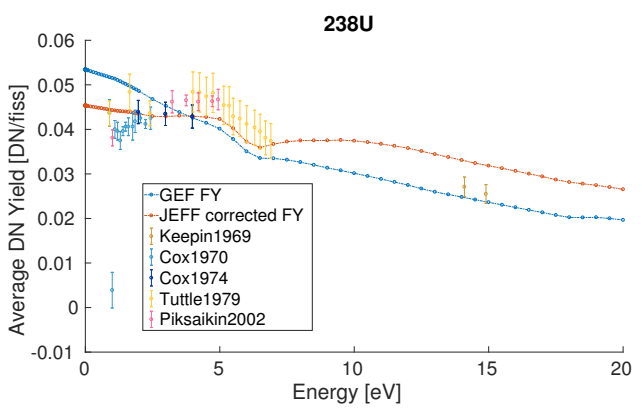

(b) $238 \mathrm{U}$

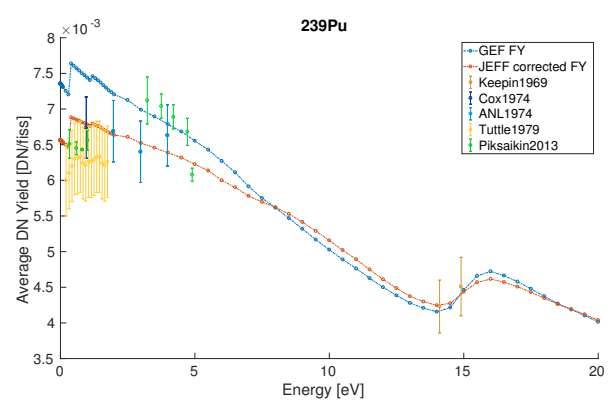

(c) $239 \mathrm{Pu}$

Figure 2: Energy dependence of average delayed neutron yield computed with GEF-6.1 FY and with JEFF-3.1.1 FY modified with GEF-6.1 energy dependence. Experimental values are reported for comparison

\subsection{Kinetic parameters and mean precursors' half-life}

A set of DN abundances is always associated to a set of decay constants and they cannot be considered separately. One way to evaluate a set of kinetic parameters $\left(a_{i}, \lambda_{i}\right)$ is to compute the derived quantity $\overline{T_{1 / 2}}$ (see Eq. 3). For the sake of clarity, instead of comparing the abundances one by one, only the effects on the $\overline{T_{1 / 2}}$ are shown and commented (see Fig.3). It seems that for ${ }^{235} \mathrm{U}$, the JEFF FY modified with GEF-6.1 better reproduce the experimental data than the original ones. The same is true for ${ }^{238} \mathrm{U}$, except at $14 \mathrm{MeV}$, where the experiments are spread and far from each other. Finally, for ${ }^{239} \mathrm{Pu}$, the trend is pretty good but a lack of measurements beyond $5 \mathrm{MeV}$ prevents us to comment on the quality of the results. Unfortunately, measurements in those energy ranges are very difficult to perform, due to the difficulty of finding a good monoenergetic neutron sources in this energy domain.

\section{Conclusions and Perspectives}

Delayed neutron data is essential in inherent reactor safety and in reactor control. The international databases rarely agree with each other, especially when DN kinetic parameters are concerned.

The $v_{d}$, the average number of delayed neutrons emitted per fission, appears in the equation of the beta effective, which is the effective fraction of neutrons causing fission 


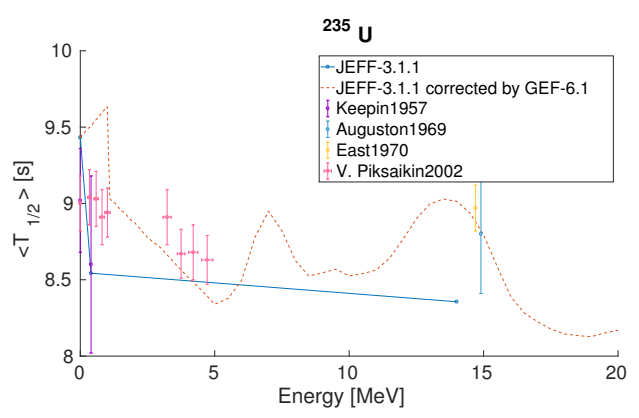

(a) $235 \mathrm{U}$

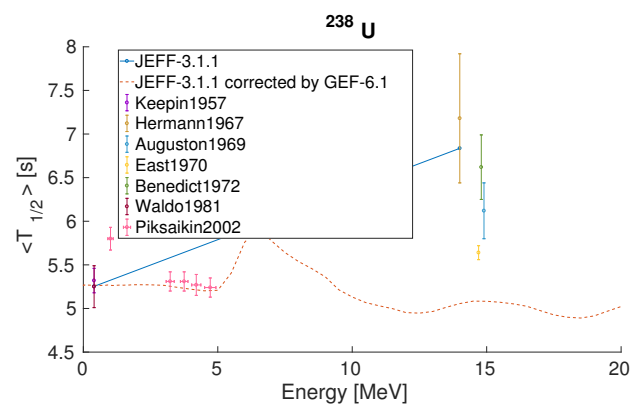

(b) $238 \mathrm{U}$

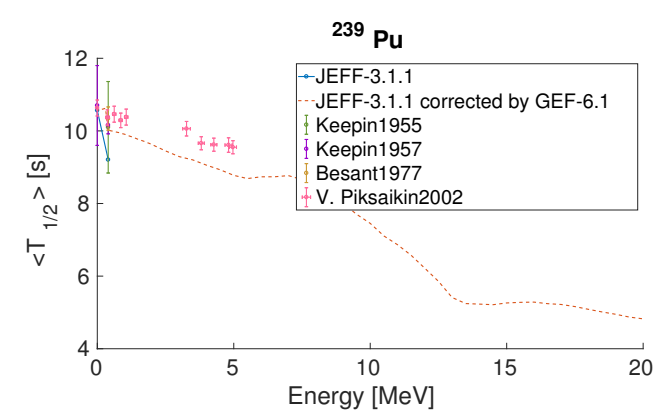

(c) $239 \mathrm{Pu}$

Figure 3: Energy dependence of the mean precursors' half-life computed with the original JEFF-3.1.1 FY and with the with JEFF-3.1.1 FY modified with GEF-6.1 energy dependence. Experimental values are reported for comparison

that are born delayed. The origin of the $v_{d}$ as a function of incident neutron energy in the international libraries is not always well reported. Comparing the evaluated values with experimental data (Fig. 2) it is clear that up to $7 \mathrm{MeV}$ and at $14 \mathrm{MeV}$ there is good agreement, while in the intermediate range ( 7 to $13 \mathrm{MeV}$ ) a lack of measurements prevents the author draw any conclusion. Applying GEF energy dependence to JEFF fission yields, the shape of the resulting $v_{d}$ presents inexplicable bumps at high energies. Furthermore, the flat behavior below $5 \mathrm{MeV}$ is not reproduced.

The the mean precursors' half-life can be estimated with the same method (Fig. 3). It seems that for ${ }^{235} \mathrm{U}$, the JEFF modified FY better reproduce the experimental data than the original ones. The same is true for ${ }^{238} \mathrm{U}$, except at $14 \mathrm{MeV}$, where the experiments are incoherent. Finally, for ${ }^{239} \mathrm{Pu}$, the agreement is acceptable until $5 \mathrm{MeV}$, beyond which a lack of measurements prevents us to infer anything on the quality of the results.

To conclude, the method presented in Section 3 is another way to evaluate the quality of fission yields. GEF-6.1 energy dependence has been tested and for the moment it is not always able to reproduce experimental data. However, the method of applying the energy dependence of a specific set of FY to another set looks promising, especially for estimating quantities of interest at energies where measurements are missing. For example, since JEFF3.1.1 gives evaluated FY at maximum 3 energies while GEF-6.1 gives calculated FY up to 20 
$\mathrm{MeV}$, the mentioned method could provide JEFF FY at almost all energies. Of course, for the method to be exploitable, GEF-6.1 should be able to reproduce JEFF-3.1.1 fission yields at energies different from the one taken as reference. For the moment this is not the case, but an improvement of GEF-6.1 energy dependence of FY could, in the future, find an application for this method.

\section{References}

[1] Energy Procedia 71, 2015, 205-212

[2] Progress in Nuclear Energy 41, 2002, 317-359

[3] Progress in Nuclear Energy 41, 2002, 391-412

[4] Los Alamos National Laboratory reports: LA-11151-MS(88), LA-11534T(89), LAUR88-4118(89)

[5] ENDF/B-VII.1 library, MT=451. The reference Lu06 appeared to be a personal communication in August 2006

[6] V. Zammit-Averlant. Validation Integrale des Estimations du Parametre Beta Effectif pour les Reacteurs MOX et Incineriteurs. PhD thesis, Université d'Aix-Marseille, 1998.

[7] Nuclear Data Sheets 131, 2016, 170-221 